\title{
CORRESPONDENCE
}

\section{To the Editor, The Mathematical Gazette}

WHEN IS A DIRECT PROOF INDIRECT?

Dear SIr.-I should like to comment on A. R. Pargeter's " direct" proof of the Steiner-Lehmus Theorem* which appeared in the February 1971 issue of the Gazette.

Let me first remark that both his proof as well as my own contribution which appeared in the December 1969 issue of the Gazette are rediscoveries of proofs which had already appeared in A. Henderson's paper in 1937 [1]. However a genuine rediscovery is made in good faith and there is nothing more to it than the unpleasant sensation it gives the rediscoverer.

It is rather the directness of the proof which I feel the obligation to challenge.

In order that a proof be direct, not only has the last stage to be proved directly but every single step has to be screened cautiously from the very beginning so as not to allow any indirect argument to slip through.

The theorem upon which Pargeter's proof is based depends very heavily on Euclid I 32, which states that the outer angle in a triangle equals the sum of the two non-adjacent inner angles; and this theorem in turn depends on Euclid I 29, stating that corresponding angles between parallels are equal. Now this last crucial theorem has no direct proof yet since Euclid's days.

We then have "...u=v and the result follows ...". How does the result follow? Apparently since the two triangles with sides $a ; l, u$ and $a, l, v$ respectively are equal, so that the original triangle has equal angles. Now it is true that equality of angles implies equality of sides but this is Euclid I 6, which again is proved indirectly.

So dropping the introduction as well as the conclusion of the proof turns out to be crucial in this case as far as directness is concerned. Apart from that it is another nice indirect proof.

It might interest readers that it was Cambridge where the directnessrequirement was first introduced in about 1850 , the time when the problem first sneaked in from the Continent. Since then many "direct" proofs have been published, so far none of them correct. Henderson alone supplied in [1] as many as seven (!) "direct" proofs (among them the one we have discussed here) all of them faulty as far as directness is concerned.

It should be quite useful for anyone about to attempt a direct proof of the Steiner-Lehmus Theorem to previously consult a paper by McBride which appeared in 1943 [2] and which concludes, quite justly as it appears, with the following highly suggestive observation: " $\ldots$ If it is held, as I hold, that Euclid I 14, Euclid I 29, Euclid I 32, and the Theorem of Pythagoras have no direct proof, then the Bisectors Theorem has not been proved directly, nor is it likely to be."

Department of Mathematics, Yours sincerely, Israel Institute of Technology, Haifa

M. LEwIN

* "If two angle bisectors in a triangle are equal, the triangle is isosceles." 


\section{REFERENCES}

1. A. Henderson: A classic problem in Euclidean Geometry. J. of the Mitchell Soc. (Dec. 1937) 246-81.

2. J. A. McBride: The equal internal bisectors theorem, 18401940. . . Many solutions or none? The Edinburgh Math. Notes, 33 (1943) $1-13$.

To the Editor, The Mathematical Gazette

THE SUM OF THREE CONSECUTIVE SQUARES

Dear Sir.-Those who were stimulated to supply proofs of my conjecture that

$$
(m+1)^{2}+(m+2)^{2}+(m+3)^{2} \quad \text { when } \quad m>0
$$

can be expressed as the sum of three other squares by use of the formulae

$$
\begin{aligned}
& (3 n-1)^{2}+(3 n)^{2}+(3 n+1)^{2}=(5 n)^{2}+(n+1)^{2}+(n-1)^{2}, \\
& (3 n)^{2}+(3 n \pm 1)^{2}+(3 n \pm 2)^{2}=(5 n \pm 2)^{2}+(n \mp 1)^{2}+n^{2},
\end{aligned}
$$

may be interested to know of another set of formulae that provides alternative sets of three squares when $m>4$ :

$$
\begin{aligned}
(9 n-1)^{2}+(9 n)^{2}+(9 n+1)^{2} & =(11 n+1)^{2}+(11 n-1)^{2}+n^{2} \\
(9 n)^{2}+(9 n \pm 1)^{2}+(9 n \pm 2)^{2} & =(13 n \pm 1)^{2}+(7 n \pm 2)^{2}+(5 n)^{2} \\
(9 n \pm 1)^{2}+(9 n \pm 2)^{2}+(9 n \pm 3)^{2} & =(11 n \pm 3)^{2}+(11 n \pm 2)^{2}+(n \mp 1)^{2} \\
(9 n \pm 2)^{2}+(9 n \pm 3)^{2}+(9 n \pm 4)^{2} & =(13 n \pm 4)^{2}+(7 n \pm 2)^{2}+(5 n \pm 3)^{2} \\
(9 n \pm 3)^{2}+(9 n \pm 4)^{2}+(9 n \pm 5)^{2} & =(13 n \pm 5)^{2}+(7 n \pm 4)^{2}+(5 n \pm 3)^{2}
\end{aligned}
$$

There are also incomplete sets of formulae that sometimes provide alternative sets :

$$
\begin{aligned}
(5 n)^{2}+(5 n \pm 1)^{2}+(5 n \pm 2)^{2} & =(7 n \pm 2)^{2}+(5 n)^{2}+(n \pm 1)^{2} \\
(5 n \pm 1)^{2}+(5 n \pm 2)^{2}+(5 n \pm 3)^{2} & =(7 n \pm 3)^{2}+(5 n \pm 2)^{2}+(n \mp 1)^{2} \\
& =(7 n \pm 2)^{2}+(5 n \pm 3)^{2}+(n \pm 1)^{2} . \\
- & \\
(7 n)^{2}+(7 n \pm 1)^{2}+(7 n \pm 2)^{2} & =(11 n \pm 2)^{2}+(5 n)^{2}+(n \mp 1)^{2} \\
(7 n \pm 1)^{2}+(7 n \pm 2)^{2}+(7 n \pm 3)^{2} & =(11 n \pm 3)^{2}+(5 n \pm 2)^{2}+(n \mp 1)^{2}
\end{aligned}
$$

From these formulae we find that

$$
\begin{aligned}
2^{2}+3^{2}+4^{2} & =0^{2}+2^{2}+5^{2}, \\
3^{2}+4^{2}+5^{2} & =0^{2}+1^{2}+7^{2}=0^{2}+5^{2}+5^{2}, \\
4^{2}+5^{2}+6^{2} & =2^{2}+3^{2}+8^{2}, \\
5^{2}+6^{2}+7^{2} & =1^{2}+3^{2}+10^{2}=2^{2}+5^{2}+9^{2}, \\
6^{2}+7^{2}+8^{2} & =1^{2}+2^{2}+12^{2}=2^{2}+8^{2}+9^{2}=0^{2}+7^{2}+10^{2}, \\
7^{2}+8^{2}+9^{2} & =3^{2}+4^{2}+13^{2}=3^{2}+8^{2}+11^{2}=1^{2}+7^{2}+12^{2} \\
& =0^{2}+5^{2}+13^{2}=5^{2}+5^{2}+12^{2}, \\
8^{2}+9^{2}+10^{2} & =2^{2}+4^{2}+15^{2}=1^{2}+10^{2}+12^{2}=0^{2}+7^{2}+14^{2}, \\
9^{2}+10^{2}+11^{2} & =2^{2}+3^{2}+17^{2}=5^{2}+9^{2}+14^{2},
\end{aligned}
$$

etc., there being always at least two different alternative sets for three consecutive squares for $m>4$.

8 Puckle Lane,

Yours faithfully,

Canterbury

Donald B. Eperson 